\title{
Integration of Quantitative Compositional Mapping and Image Processing Routines: A Powerful Approach to Petrologic Investigations
}

\author{
T.M. Hahn ${ }^{1}$, P.K. Carpenter ${ }^{1}$ and B.L. Jolliff ${ }^{1}$ \\ ${ }^{1}$. Department of Earth and Planetary Science, McDonnell Center for the Space Sciences, Washington University \\ in St. Louis (WUSTL), Campus Box 1169, St. Louis, MO, 63130, USA
}

The petrology- and mineralogy-oriented communities have long recognized the need for the integration of quantitative chemical analyses with qualitative observations (e.g., textural analysis). In response, numerous efforts have focused on the integration of geochemical datasets and image processing software to extract and interpret geochemical information, while retaining textural relationships; however, only moderate success has been achieved, as applications have generally been limited to simple systems that contain relatively few mineral phases with homogeneous chemical compositions, and a long data-acquisition time is required [e.g., 1,2]. We present the first integration of fully quantitative electron probe microanalysis (EPMA) compositional mapping with remote sensing software Environment for Visualizing of Images (ENVI) and IDL Workspace. Our methods provide a powerful tool for petrologic investigations and alleviate current issues with similar techniques [i.e., 2]. We demonstrate applications to relevant petrologic problems, using mineralogic and lithologic mapping procedures, and discuss the relevant implications to other fields (i.e. mineral science). Detailed documentation of data and image analysis procedures will be made available, upon request and alongside submission to scholarly journals, in the form of IDL scripts (data analysis and visualization) and ENVI tutorials (image processing and visualization). Our goal is to provide a new, easily accessible, tool for the interrogation of geologic materials.

We present compositional, mineralogical, and lithological distribution maps of complex meteorite breccia Northwest Africa (NWA) 2995 (Fig. 1) to demonstrate the applicability of our newly developed data and image analysis techniques. Compositional stage mapping procedures and data collection processes are discussed in [3]; therefore, only a summary is presented here. Quantitative compositional mapping was conducted using the JEOL JXA-8200 electron microprobe at WUSTL; JEOL and Probe Software was used to acquire backscattered-electron (BSE) and X-ray intensity maps. The generated elemental maps typically comprises a suite of $>10$ elements with a full matrix correction applied, which includes a mean atomic number background correction, and results in a complete quantitative analysis (wt. \% element) at each pixel. Output data for a single element is processed and converted to an image format suitable for ENVI using IDL Workspace. Elemental composition images are combined in ENVI to produce a geochemical information cube, analogous to a multispectral image cube. In this representation, a single band within a composite image, containing all of the quantitative compositional information, represents a single element. Using a band threshold procedure, all analyses with undesirable statistics (e.g., totals $<98 \%$ and $>102 \%$ ) and cracks within the sample area can be selected, and a mask created, in order to eliminate analyses from the dataset that do not meet specified criteria. Depending on the investigation objectives, regions of interest (ROIs) can be created to parse data into subsets (e.g., individual clasts and the matrix in complex breccias), which allows for the analysis and statistical treatment of the selected data separately.

We applied a supervised classification procedure in ENVI (i.e., minimum distance in n-dimensional chemical space) to classify the mineralogy of the NWA 2995 (e.g., bulk sample, matrix, and individual clasts; Table 1; Figs. 2 and 3). Supervised classification schemes require user input in the form of a "training set." We identified phases based on the element data (wt. \% element), and selected ROIs accordingly, to develop a training set for classification routines. We selected phase ROIs on a clast-by-clast basis to elucidate key features in the matrix mineralogy (e.g., from which clasts are mineral fragments derived?). Classifications are performed iteratively, where in each successive classification, phases that are unclassified (i.e., compositionally distinct from other classified phases) are used to generate new ROIs and classification routines are iterated until the total unclassified pixels is $<5 \%$. We also explore additional supervised, as well as unsupervised, classification routines. The resulting classification images are used to extract the processed wt. \% element data by mineralogy, clast type, and 
mineral fragments derived from the matrix (Table 1; Fig. 2). Using ENVI protocols and IDL routines, images are generated, which contain information regarding stoichiometry, mineral end-member compositions, elemental ratios, phase distribution, and fracture porosity. Additionally, average mineral compositions are calculated; density-corrected bulk composition reconstructions are performed on lithic clasts, matrix components, and exsolved or zoned minerals (Table 1); and variation diagrams are constructed.

We have developed a highly effective method for petrologic investigations. Specifically, our analytical techniques and data processing routines are well suited for investigation of complex samples and for which only limited quantities exist (e.g., meteoritic and Apollo breccias). Investigations that require high precision and accuracy (i.e., resource exploration) are also well suited. The integration of high-quality EPMA compositional mapping with remote sensing software ENVI, coupled with IDL Workspace, allows for continued downstream developments, and offers easy integration of additional capabilities. Additionally, ENVI and IDL Workspace are typically easily accessible within most geology departments.

\section{References:}

[1] Maloy and Trieman, Am. Min. 92 (2007) p. 1781.

[2] Pret et al., Am. Min. 95 (2010) p. 1379.

[3] Carpenter et al., Microsc. Microanal. (2017).

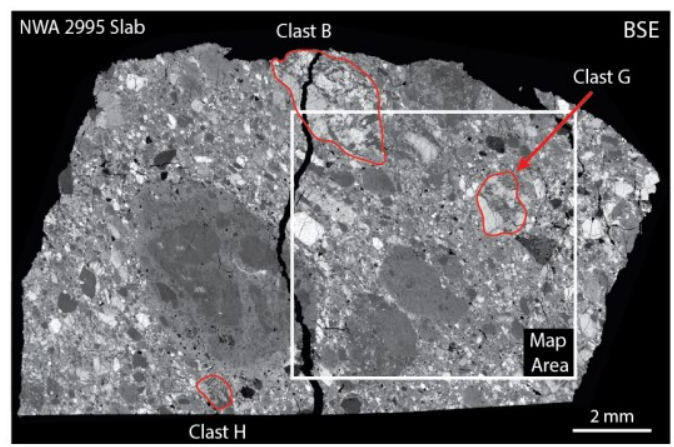

Figure 1. Backscattered electron image, displaying the location of mapping areas and lithic clasts.

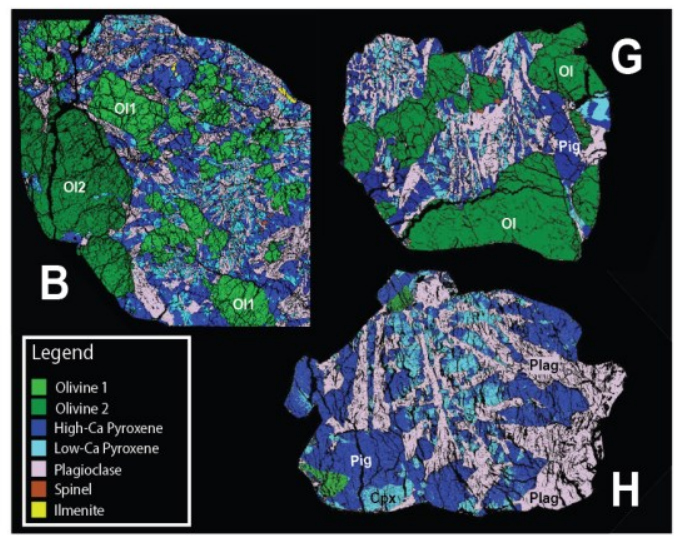

Figure 2. High spatial resolution classification images of lithic clasts in NWA 2995. Lithic clast data is isolated, and used to calculate density-corrected bulk composition; a similar routine is applied to the matrix component of the breccia (Table 1).
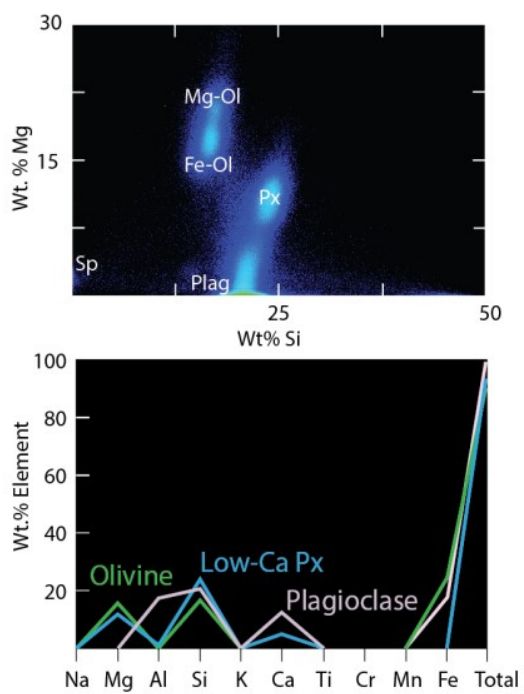

Figure 3. Element variation diagram used for the discrimination of mineral phases. Also shown is a "chemical spectrum" used to further identify phases. In this representation, the "chemical spectrum" is analogous to a X-ray spectrum.

Table 1. Calculated bulk composition of selected regions. (wt.\% oxide)

\begin{tabular}{|c|c|c|c|c|c|c|c|c|c|c|c|}
\hline \multirow[b]{2}{*}{ Oxide } & \multirow{2}{*}{$\begin{array}{c}\text { Fused } \\
\text { Bead }\end{array}$} & \multirow{2}{*}{ Matrix } & \multicolumn{3}{|c|}{ Clasts } & \multirow{2}{*}{\multicolumn{2}{|c|}{$\begin{array}{c}\text { Fused } \\
\text { Bead }\end{array}$}} & \multirow{2}{*}{ Matrix } & \multicolumn{3}{|c|}{ Clasts } \\
\hline & & & B & G & $\mathrm{H}$ & & & & B & G & $\mathrm{H}$ \\
\hline $\mathrm{SiO}_{2}$ & 46.2 & 46.3 & 43.4 & 43.6 & 49.6 & $\overline{\mathrm{Mg}}$ \# & 59.6 & 60.0 & 62.7 & 66.8 & 69.5 \\
\hline $\mathrm{TiO}_{2}$ & 0.68 & 0.53 & 0.42 & 0.22 & 0.29 & En & & & 45 to 54 & 48 to 60 & 49 to 60 \\
\hline $\mathrm{Al}_{2} \mathrm{O}_{3}$ & 20.6 & 21.4 & 7.44 & 6.96 & 15.1 & Wo & & & 14 to 33 & 13 to 35 & 13 to 33 \\
\hline $\mathrm{Cr}_{2} \mathrm{O}_{3}$ & 0.23 & 0.2 & 0.31 & 0.34 & 0.38 & An & & & 91 & 89 & 90 \\
\hline MgO & 8.08 & 8.11 & 19.8 & 22.3 & 12.8 & $\mathbf{A b}$ & & & 9 & 10 & 9 \\
\hline $\mathrm{CaO}$ & 13.5 & 13.1 & 7.24 & 6.53 & 12.8 & & & & & & \\
\hline MnO & 0.15 & 0.13 & 0.27 & 0.26 & 0.19 & Modz & a Analy & & & & \\
\hline $\mathrm{FeO}^{\top}$ & 9.75 & 9.65 & 21 & 19.8 & 10 & Fo & & & 58 to 68 & 65 & 66 \\
\hline $\mathrm{Na}_{2} \mathrm{O}$ & 0.51 & 0.6 & 0.22 & 0.22 & 0.44 & Plag & & & 22.8 & 21.3 & 44.4 \\
\hline $\mathrm{K}_{2} \mathrm{O}$ & 0.19 & 0.15 & 0.02 & 0.03 & 0.05 & Ol-1 & & & 22.7 & 47.6 & 1.68 \\
\hline \multirow[t]{5}{*}{ Total } & 99.9 & 100.2 & 100.1 & 100.3 & 101.7 & Ol-2 & & & 18 & & \\
\hline & & & & & & High- & Ca Px & & 11 & 10.2 & 15.8 \\
\hline & & & & & & Low-C & Ca Px & & 25.1 & 20.7 & 38.2 \\
\hline & & & & & & $\mathrm{Ilm}$ & & & 0.2 & & \\
\hline & & & & & & Sp & & & 0.36 & 0.27 & \\
\hline
\end{tabular}

